The effect of unilateral hand contractions on psychophysiological activity during motor performance: Evidence of verbal-analytical engagement Hoskens, Merel; Bellomo, Eduardo; Uiga, Liis; Cooke, Andrew; Masters, Rich

\title{
Psychology of Sport and Exercise
}

DOI:

10.1016/j.psychsport.2020.101668

Published: 30/05/2020

Peer reviewed version

Cyswllt i'r cyhoeddiad / Link to publication

Dyfyniad o'r fersiwn a gyhoeddwyd / Citation for published version (APA):

Hoskens, M., Bellomo, E., Uiga, L., Cooke, A., \& Masters, R. (2020). The effect of unilateral hand contractions on psychophysiological activity during motor performance: Evidence of verbalanalytical engagement. Psychology of Sport and Exercise, 48, [101668].

https://doi.org/10.1016/j.psychsport.2020.101668

\footnotetext{
Hawliau Cyffredinol / General rights

Copyright and moral rights for the publications made accessible in the public portal are retained by the authors and/or other copyright owners and it is a condition of accessing publications that users recognise and abide by the legal requirements associated with these rights.

- Users may download and print one copy of any publication from the public portal for the purpose of private study or research.

- You may not further distribute the material or use it for any profit-making activity or commercial gain

- You may freely distribute the URL identifying the publication in the public portal ?
}

Take down policy

If you believe that this document breaches copyright please contact us providing details, and we will remove access to the work immediately and investigate your claim. 


\section{Journal Pre-proof}

The effect of unilateral hand contractions on psychophysiological activity during motor performance

Merel C.J. Hoskens, Eduardo Bellomo, Liis Uiga, Andrew Cooke, Rich S.W. Masters

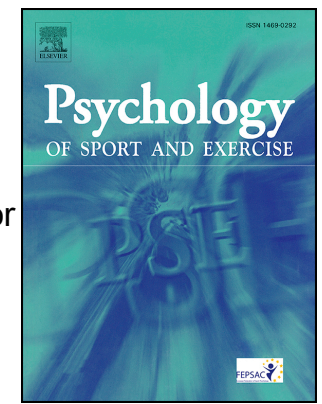

PII:

S1469-0292(19)30778-2

DOI:

https://doi.org/10.1016/j.psychsport.2020.101668

Reference: PSYSPO 101668

To appear in: Psychology of Sport \& Exercise

Received Date: 12 November 2019

Revised Date: 23 January 2020

Accepted Date: 9 February 2020

Please cite this article as: Hoskens, M.C.J., Bellomo, E., Uiga, L., Cooke, A., Masters, R.S.W., The effect of unilateral hand contractions on psychophysiological activity during motor performance,

Psychology of Sport \& Exercise (2020), doi: https://doi.org/10.1016/j.psychsport.2020.101668.

This is a PDF file of an article that has undergone enhancements after acceptance, such as the addition of a cover page and metadata, and formatting for readability, but it is not yet the definitive version of record. This version will undergo additional copyediting, typesetting and review before it is published in its final form, but we are providing this version to give early visibility of the article. Please note that, during the production process, errors may be discovered which could affect the content, and all legal disclaimers that apply to the journal pertain.

(C) 2020 Published by Elsevier Ltd. 
Merel Hoskens: Conceptualization, methodology, formal analysis, investigation, writing original draft, visualization,

Eduardo Bellomo: Methodology, formal analysis, investigation, writing - review \& editing Liis Uiga: Conceptualization, methodology, writing - review \& editing and supervision Andrew Cooke: Conceptualization, methodology, formal analysis, writing - review \& editing and supervision

Rich Masters: Conceptualization, methodology, formal analysis, writing - review \& editing and supervision 
The effect of unilateral hand contractions on psychophysiological activity during motor performance

Merel C.J. Hoskens ${ }^{I *}$, Eduardo Bellomo ${ }^{2}$, Liis Uiga ${ }^{l}$, Andrew Cooke $^{2} \&$ Rich S.W. Masters ${ }^{l}$

1: Te Huataki Waiora School of Health, University of Waikato, NZ

2: School of Sport, Health \& Exercise Sciences, Bangor University, UK

Corresponding author*:

\author{
Merel Hoskens \\ School of Health \\ University of Waikato
}

Private Bag 3105, Hamilton 3240, New Zealand

E: mcjhh1@students.waikato.ac.nz 


\begin{abstract}
Objectives: Conscious engagement in movement control can influence motor performance. In most cases, the left hemisphere of the brain plays an important role in verbal-analytical processing and reasoning, so changes in the balance of hemispheric activation may influence conscious engagement in movement. Evidence suggests that unilateral hand contractions influence hemispheric activation, but no study has investigated whether there is an associated effect of hand contractions on verbal-analytical processing and psychophysiological activity during motor performance. This study was designed to examine whether pre-performance unilateral hand contraction protocols change verbal-analytical involvement and psychophysiological activity during motor performance. Design: A repeated measures crossover design was employed. Methods: Twenty-eight participants completed three hand contraction protocols in a randomised order: left, right and no-hand contractions. Electroencephalography (EEG) measures of hemispheric asymmetry were computed during hand contractions. A golf putting task was conducted after each protocol. EEG connectivity between sites overlying the left verbal-analytical temporal region (T7) and the motor planning region $(\mathrm{Fz})$ was computed for the 3 -sec prior to movement initiation. Additionally, electrocardiography (ECG) and electromyography (EMG) signals were analysed 6-sec prior to movement initiation until 6-sec after. Golf putting performance was obtained by distance from the target and putter swing kinematics. Results: Contralateral hemisphere activity was revealed for the left and right-hand contraction conditions. During motor planning, the lefthand contraction protocol led to significantly lower T7-Fz connectivity, and the right-hand contraction protocol led to significantly higher T7-Fz connectivity than the other conditions. EMG, ECG and kinematic measures did not differ as a function of condition. Importantly, T7-Fz connectivity mediated the relationship between hand squeezing and motor performance (distance from the target). Conclusion: The EEG results suggest that pre-
\end{abstract}




\section{Journal Pre-proof}

performance unilateral hand contractions influence the extent of verbal-analytical engagement in motor planning, which in turn influences motor performance. However, the hand contractions did not influence cardiac activity, muscle activity or kinematics.

Key words: hand contraction protocol; hemisphere-specific priming; EEG; heart rate; movement kinematics 


\section{Introduction}

A link between conscious processes and motor performance is found in studies using

3 electroencephalography (EEG) to examine communication (synchronization) between

4 different regions of the brain (Babiloni et al., 2011; Deeny, Hillman, Janelle, \& Hatfield,

5 2003; Gallicchio, Cooke, \& Ring, 2016; Zhu, Poolton, Wilson, Maxwell, \& Masters, 2011).

6 Evidence from these studies suggests that high conscious engagement in motor performance

7 is associated with more synchronous neuronal activity, indexing greater functional communication between the left temporal T7 region of the brain (involved in verbalanalytical processing), and the frontal midline Fz region of the brain (involved in motor planning) (Babiloni et al., 2011; Deeny et al., 2003; Gallicchio et al., 2016; Zhu et al., 2011). Compelling evidence for the link between conscious control of movements and verbal-analytical processes has been reported by Zhu et al. (2011, Experiment 1). They measured propensity to consciously control motor skills using the Movement Specific Reinvestment Scale (MSRS, Masters, Eves, \& Maxwell, 2005). Participants with a lower propensity to consciously control movements displayed lower T7-Fz communication (e.g., coherence) than participants with a higher propensity for conscious control, during the 4-sec preceding golf putts (Zhu et al., 2011). Co-activation between the left temporal and frontal regions is also associated with motor performance. For example, Gallicchio et al. (2016) reported that T7-Fz connectivity was lower in the final seconds preceding successful golf putts compared to unsuccessful golf putts, suggesting that reduced or suppressed verbalanalytical processing is a feature of effective motor performance. In sum, reduced left temporal-frontal synchronicity may be associated with less verbal, more procedural, processing of movements.

Attempts to reduce verbal-analytical engagement during motor performance have used neuro-stimulation to suppress activity in the left hemisphere (Landers et al., 1991; 
Snyder et al., 2003; Zhu et al., 2015). For instance, Zhu et al. (2015) found that cathodal (i.e., inhibitory) transcranial Direct Current Stimulation (tDCS) over the left dorsolateral prefrontal cortex promoted lower verbal-analytical engagement when practicing a golf putting task, compared to sham stimulation (i.e., placebo). However, tDCS is not a practical or accessible training method for the majority of performers, and ethical concerns about such extreme training methods have been raised (Davis, 2013).

Gröpel and Beckmann (2017) asked semi-professional athletes (gymnastics, soccer,

badminton and taekwondo) to squeeze a stress ball in either the left hand or the right hand for 45-sec before performing under competitive pressure. They reasoned that due to the contralateral coupling between our hands and our brain (i.e., the brain area controlling the right hand resides in left hemisphere, and vice-versa), squeezing the right hand should prime the left (verbal-analytic) hemisphere and squeezing the left hand should prime the right (visual-spatial) hemisphere. Results showed that left-hand contractions resulted in more stable performance under pressure than right-hand contractions. The authors argued that lefthand contractions prevented breakdown under pressure by activating the right hemisphere and deactivating the left hemisphere, which reduced disruptive verbal-analytical control of the movements (Beckmann et al., 2013; Gröpel \& Beckmann, 2017). Beckmann et al. (2013, Experiment 3) additionally found that right-hand contractions magnified the effect of pressure, with participants performing worse when they carried out right-hand contractions prior to performing. They suggested that since right-hand contractions activated the left hemisphere, they potentially increased the likelihood that pressure would cause disruptive verbal-analytical involvement in performance. However, it is important to note that this interpretation cannot be confirmed since Beckmann and colleagues did not directly measure 50 cortical activity in their studies. 
Studies that did record cortical activity during unilateral hand contractions have

revealed inconsistent results. For example, some studies revealed that unilateral hand

contractions result in lower alpha power (i.e., increased brain activity) in the contralateral

hemisphere (Gable, Poole, \& Cook, 2013; Harmon-Jones, 2006; Peterson, Shackman, \&

Harmon-Jones, 2008; Schiff, Guirguis, Kenwood, \& Herman, 1998). However, Cross-

Villasana, Gropel, Doppelmayr, and Beckmann (2015) revealed that unilateral hand

contractions produced lower alpha power over both hemispheres. Furthermore, they revealed

that immediately after left-hand contractions ceased, whole scalp alpha power increased,

indicating widespread deactivation (Cross-Villasana et al., 2015). This latter finding

challenges Beckmann and colleagues suggestion that left-hand contractions are beneficial

because they activate the right hemisphere. However, it does support the argument that left-

hand contractions can deactivate the left hemisphere, perhaps suppressing verbal-analytical

engagement in motor planning. Taken together, these findings indicate that hemispheric analytical processes have yet to be established. Specifically, no study has examined the effect of unilateral hand contractions on T7-Fz connectivity during the final moments of motor preparation. These final moments are important for establishing the level of conscious monitoring and control of the movement (e.g., Deeny et al., 2003; Gallicchio et al., 2016; Zhu et al., 2011). Therefore, measurement of cortical activity, especially T7-Fz connectivity, is required to more rigorously examine the proposed relations between left-hand contractions, verbal-analytical engagement and motor performance.

Finally, no studies have investigated the effects of hand contraction protocols on physiological and kinematic measures that may also relate to verbal-analytical engagement and motor performance outcomes (Cooke, Kavussanu, McIntyre, \& Ring, 2010). Although 
deceleration during the 6-sec preceding motor performance in skilled versus low skilled golfers. Therefore, heart rate deceleration could offer another corroborative physiological measure that is sensitive to the amount of verbal-analytical engagement during motor planning (Cooke et al., 2014; Neumann \& Thomas, 2009; Neumann \& Thomas, 2011; Radlo, Steinberg, Singer, Barba, \& Melnikov, 2002). Similarly, more automatic motor control is also associated with lower muscle activity (Lohse, Sherwood, \& Healy, 2010; Vance, Wulf, Tollner, McNevin, \& Mercer, 2004; Zachry, Wulf, Mercer, \& Bezodis, 2005). For example, Lohse et al. (2010) revealed lower muscle activity when participants adopted an external focus of attention while throwing darts, compared to when they consciously monitored their technique. Finally, movement kinematics can also be linked to verbal-analytical engagement in motor planning (Cooke et al., 2014; Malhotra, Poolton, Wilson, Omuro, \& Masters, 2015; Masters, Poolton, Maxwell, \& Raab, 2008; Maxwell, Masters, \& Eves, 2003). For example, Maxwell et al. (2003) revealed that verbal-analytic engagement in motor planning was associated with a less fluid technique. The assessment of such measures alongside T7-Fz connectivity may therefore provide new insight into the mechanisms underpinning the effects of unilateral hand contraction protocols on performance.

The present study is the first to investigate the effect of unilateral hand contraction protocols on psychophysiological and behavioural markers of golf putting performance. The aim was to gain a better understanding of whether pre-performance unilateral hand contractions have an effect on verbal-analytical processes involved in motor performance. Three hand contraction protocols (left, right and no-hand) were performed in a repeated measures crossover design, before performance of a golf putting task. Measures of alpha power $(8-12 \mathrm{~Hz})$ between homologous electrode pairs were first computed during the hand contraction protocols to verify that left-hand contractions activated the right hemisphere, and that right-hand contractions activated the left hemisphere. Cortical activity was then 
101 examined further by measuring the high-alpha power $(10-12 \mathrm{~Hz})$ connectivity level between

102 the verbal-analytical left temporal (T7) region and the motor planning (Fz) region during

103 preparation for each golf putt. Cardiac activity (electrocardiography), muscle activity

104 (electromyography), kinematics, and golf performance were tested as supporting measures of

105 verbal-analytical engagement in motor planning. Mediation analyses were employed to

106 examine whether our EEG and psychophysiological indices of verbal-analytic engagement

107 are the mechanisms underpinning any effect of hand contractions on performance.

108 Based on the behavioural findings of Beckmann et al. (2013) and Gröpel and

109 Beckmann (2017), we predicted that unilateral hand contractions would influence verbal-

110 analytical involvement (i.e., inferred by changes in T7-Fz connectivity) during movement

111 planning. Specifically, we predicted that the left-hand contractions would lower verbal-

112 analytical involvement during motor planning compared to right-hand and no-hand

113 contractions, and that right-hand contractions would raise verbal-analytical involvement in

114 motor planning compared to left-hand and no-hand contractions. Consequently, lower verbal-

115 analytical engagement during the left-hand contraction protocol was expected to promote

116 greater heart rate deceleration, lower muscular activity, smoother kinematics when initiating

117 the golf putt and better outcome performance compared to the right-hand and no-hand

118 contraction protocols (Cooke et al., 2014; Lohse et al., 2010; Neumann \& Thomas, 2009;

119 Radlo et al., 2002; Zachry et al., 2005). The opposite effects were predicted for the right-hand

120 contraction protocol. Finally, we predicted that the effects of hand contractions on $\mathrm{T} 7 \mathrm{-Fz}$

121 connectivity and our ECG, EMG and kinematic measures would mediate the relationship

122 between hand contraction protocols and performance. 


\section{Methods}

\section{Participants and design}

Twenty-eight people were recruited to participate in the experiment. Three

126 participants who had major artefacts in their EEG signal were excluded from further analysis,

127 resulting in a final sample of twenty-five participants (mean age $=26.52, \mathrm{SD}=5.08$, female $=$

128 15). To control for handedness, only right-handed participants were included (> 70,

129 Edinburgh Handedness Inventory, Oldfield, 1971). All participants had normal/corrected

130 vision. The participants were instructed not to consume alcohol or drugs 24-hours prior to

131 testing or caffeine 3-hours prior to testing, and to obtain at least 6-hours of sleep the night

132 before testing. A repeated measures crossover design was adopted, with participants

133 performing three different protocols (right, left and no-hand contractions). The order of

134 protocols was counterbalanced within participants. This study was approved by the

135 University (Human) Research ethics committee.

136 Task

137 The experiment consisted of a pre-performance hand contraction protocol followed by

138 a golf putting task. The hand contraction protocol required participants to firmly contract a

139 stress ball at a self-paced rate for 45-sec either with their left hand or right hand, or to place

140 their hands on their lap and hold them still for 45-sec (no-hand contraction condition). The

141 researcher instructed the participants to sit quietly and to not talk or make large movements

142 during these protocols, in order to control for muscle activity artefacts.

143 After each protocol, participants performed 25 golf putts on an artificial grass surface, 144 using a standard length $(90-\mathrm{cm})$ golf putter and a regular-size (diameter $4.7-\mathrm{cm})$ golf ball.

145 The target was a 1-cm diameter white sticker on the putting surface positioned 2.4-m from

146 the initial starting point. Mean radial error (mean distance in any direction from the target)

147 was assessed. 
Measures

\section{Psychophysiological measures.}

EEG data was used to assess cortical activity during the pre-performance hand

contraction protocols (e.g., Gable et al., 2013) and during preparation of the golf putts (e.g.,

152 Zhu et al., 2011). EEG was recorded from thirty-two (32) active electrodes positioned using

153 the 10-20 system (Jaspers, 1958): Fp1, Fp2, AF3, AF4, F7, F3, Fz, F4, F8, FC5, FC1, FC2,

FC6, T7, C3, Cz, C4, T8, CP5, CP1, CP2, CP6, P7, P3, Pz, P4, P8, PO3, PO4, O1, Oz, and

O2. Additionally, active electrodes were positioned on each mastoid, at the outer canthus and

below each eye to record vertical and horizontal electrooculogram (EOG). Monopolar

recorded signals were sampled at $1024 \mathrm{~Hz}$, without an online filter, using an ActiveTwo amplifier (Biosemi, The Netherlands).

During the pre-performance protocols, we were primarily interested in cortical asymmetry (i.e., right hemisphere minus left hemisphere) in the broad alpha band frequency (i.e., $8-12 \mathrm{~Hz}$ ), as previous studies have demonstrated the effects of unilateral hand contractions on broad-band alpha (Cross-Villasana et al., 2015; Gable et al., 2013; HarmonJones, 2006; Peterson et al., 2008). During preparation of the golf putt, we were interested in connectivity in the high-alpha frequency band (i.e., 10-12 Hz), as this portion of the alpha frequency is thought to be specifically related to task specific attentional processes and cortico-communication (Smith, McEvoy, \& Gevins, 1999; for a review see Klimesch, 1999). cardiac activity (Cooke et al., 2014; Cooke, Kavussanu, McIntyre, Boardley, \& Ring, 2011).

169 Silver/silver chloride spot electrodes (BlueSensor SP, Ambu, Cambridgeshire, UK) were placed on each clavicle and on the lowest left rib. The ECG signal was amplified (Bagnoli-4, 
172 (CED Power 1401, Cambridge Electronic Design, Cambridge, UK) using Spike2 software

173 (version 5, Cambridge Electronic Design).

174 Electromyography (EMG) was used to obtain muscle activity during golf putting for

175 the extensor carpi radialis and flexor carpi ulnaris muscles in the left arm (Cooke et al., 2014;

176 Cooke et al., 2011). Differential surface electrodes (DE 2.1, Delsys) were placed on the belly

177 of the muscles and a ground electrode (BleuSensor SP, Ambu, Cambridgeshire, UK) was

178 placed on the left collarbone. The EMG signal was amplified (Bagnoli-4, Delsys), filtered

179 (20-45 Hz), and digitized at $2500 \mathrm{~Hz}$ with 16-bit resolution (Power 1401) using Spike2

180 software.

$181 \quad$ Golf putting performance measures.

182 The golf putting performance was determined by the mean radial error $(\mathrm{cm})$,

183 representing the mean distance between the final position of the ball and the centre of the

184 target. This measure was computed with ScorePutting software (written in National

185 Instruments LabVIEW), which uses the photographs from a camera system directly placed

186 above the targets to control for angle differences (Neumann \& Thomas, 2008).

$187 \quad$ Golf kinematics.

188 A triaxial accelerometer (LIS3L06AL, ST Microelectronics, Geneva, Switzerland)

189 and amplifier (frequency response of DC to $15 \mathrm{~Hz}$ ) were attached to the rear of the putter

190 head in order to measure movement kinematics (Cooke et al., 2014; Cooke et al., 2011).

191 Acceleration of the golf putter from downswing until ball contact was calculated for the $\mathrm{x}, \mathrm{y}$

192 and z-axes (representing the lateral, vertical and back-and-forth movement of the club head),

193 to determine club head orientation, swing height and impact force (Spike2, version 5,

194 Cambridge Electronic Design). 
Procedure

Participants were informed about the context of the study and signed an informed consent form prior to the start of the experimental procedure. The EEG, ECG and EMG equipment were set up and a 2-min EEG resting state measurement was performed (1-min open eyes and 1-min closed eyes).

Participants first completed 130 putts as part of a separate investigation of the psychophysiological corollaries of practice (data not reported here). The putts served to familiarise participants with the task. This was followed by performing one of the three preperformance hand-contraction protocols (left, right or no-hand contractions) while seated.

Immediately after each protocol, participants were instructed to stand-up and perform 25 selfpaced golf putts, aiming for the target as accurately as possible. The time lag between the end of the squeezing protocol and the start of the putting task was approximately 10-sec. A photograph of the final position of the golf ball was taken after each trial. The researcher then collected the golf ball and positioned it for the next trial, thereby standardising the inter-trial interval, and reducing the need for participants to move in-between putts. This procedure was

210 repeated for all conditions (three times in total) and took on average 5-min and 53-sec per 211 condition.

\section{Analysis}

\section{Pre-performance hand contraction protocols.}

EEG signals captured during the hand contraction protocols were processed offline with EEGLAB software (Delorme \& Makeig, 2004) running on MATLAB (Mathwork, Inc.,

216 USA version 2018b) to compute the power asymmetry. The signals were first resampled to

$217250 \mathrm{~Hz}$, re-referenced to the average of all electrodes, and filtered (.01-30 Hz bandpass filter).

218 The IAF toolbox was used to adjust the alpha frequency band for each participant based on 
219 their individual alpha frequency peak, determined from the baseline measure (Corcoran,

220 Alday, Schlesewsky, \& Bornkessel-Schlesewsky, 2018).

The signals were then subjected to a threshold-based artefact removal procedure,

222 where any 250 -ms window containing signal fluctuations exceeding $\pm 150 \mu \mathrm{V}$ was rejected

223 (ERPLAB Toolbox, Lopez-Calderon \& Luck, 2014). Independent Component Analyses were then performed via the RunICA infomax algorithm (Makeig, Bell, Jung, \& Sejnowski, 1996)

to identify and remove any remaining artefacts and non-neural activity (e.g., eye-blinks) from the signal. An average of 5.76 components were rejected. The clean signal was then subjected to a time frequency analysis, to obtain the estimate of instantaneous alpha power for the 38sec of the hand contraction protocols. The total of $45-\mathrm{sec}$ was reduced by $7-\mathrm{sec}$, due to some participants showing increased artefacts at the end. This analysis was performed by convolving the Fast-Fourier Transform (FFT) power spectrum of the signal with a family of complex Morlet wavelets and eventually taking the inverse FFT (Cohen, 2014). All power

232 values were then log transformed to control for skewness and inter-individual differences.

233 Finally, the transformed values were used to compute the asymmetry scores of the

234 homologous electrode pairs close to the cortical regions involved in hand movements (e.g.,

235 Grefkes, Eickhoff, Nowak, Dafotakis, \& Fink, 2008): T8-T7, P4-P3, P8-P7, F4-F3, F8-F7,

236 C4-C3, FC2-FC1, FC6-FC5, CP2-CP1, CP6-CP5 (right - left). This is a common way of

237 calculating alpha asymmetry to identify the effects of a state manipulation (e.g., unilateral

238 hand contractions) on the relative activation of the right hemisphere versus left hemisphere of

239 the brain (e.g., Harmon-Jones, 2006). A higher asymmetry score signifies more activity in the

240 left hemisphere (inverse of alpha activity) compared to the right hemisphere (Harmon-Jones,

241 2006; Wolf et al., 2015). 
Golf putting task.

An optical sensor and microphone were used to mark movement initiation and ball

244 contact in the continuous data (Spike2 and Actiview software, Biosemi), in order to analyse

245 the psychophysiological measures prior to and during the golf putts. The optical sensor (S51-

246 PA-2-C10PK, Datasensor, Monte San Pietro, Italy) was used to identify swing-onset by

247 detecting when the infrared beam was broken by movement of the putter head. The microphone (NT1, Rode, Silverwater, Australia) was linked to a mixing desk (Club 2000, Studiomaster, Leighton Buzzard, UK) to detect putter-to-ball contact.

Connectivity prior to movement initiation was computed offline by processing the

EEG signals (EEGLAB software) computed during the golf putt preparation. The signals were cut into epochs of 5-sec (4-sec prior to and 1-sec after movement initiation). Thereafter, the signals were filtered and cleaned with the same methods as for the hand contraction protocols. The signals were then baseline corrected $(-.2$ to 0 -sec, where $0=$ movement initiation; Ring et al., 2015) and time-frequency analysis was performed (see hand contraction protocols) to obtain the phase angles. These phase angles were then used to compute connectivity between the left temporal (T7) and frontal (Fz) regions for the 3 -sec prior to movement initiation, by calculating inter-site phase clustering (ISPC, Cohen, 2014). ${ }^{1}$ We calculated ISPC $_{\text {time }}$ measuring phase angle differences across the electrodes over time: ${ }^{2}$

$$
\operatorname{ISPC}_{x y}(f)=\left|n^{-1} \sum_{t=1}^{n} e^{i\left(\theta_{x}(t f)-\theta_{y}(t f)\right)}\right|
$$

\footnotetext{
${ }^{1}$ Two different methods have been used to measure synchronization in the sport science literature.
} Earlier work (e.g., Deeny et al., 2003) measured magnitude squared coherence; however, more recent research has measured inter-site phase connectivity (ISPC). ISPC is based on phase information only, which makes it independent of fluctuations in absolute power (Gallicchio et al., 2016).

${ }^{2}$ Cohen (2014) suggests that the ISPC time measure is appropriate when having relatively long epochs, with 3-sec considered as long. 
$N$ is the number of data points; $i$ is the imaginary operator; $\theta_{x}$ and $\theta_{y}$ are the phase angles of

261 the recorded signal at two different scalp locations; $t$ is the time point and $f$ is the frequency

bin. The $\mathrm{e}^{\mathrm{i}\left(\theta_{\mathrm{x}}(\mathrm{tf})-\theta_{\mathrm{y}}(\mathrm{tf})\right)}$ represents the complex vector with magnitude 1 and angle $\theta_{x}-\theta_{y}$;

$263 \mathrm{n}^{-1} \sum_{\mathrm{t}=1}^{\mathrm{n}}($.$) denotes averaging over time points, and |$.$| is the module of the averaged vector$

264 (Cohen, 2014; Lachaux, Rodriguez, Martinerie, \& Varela, 1999). ISPC is given as a value between 0 (no functional connection) and 1 (perfect functional connection). Finally, values were Z-transformed (inverse hyperbolic tangent) to ensure normal distribution (Gallicchio et al., 2016).

The EMG and ECG signals 6-sec prior to until 6-sec after movement initiation were analysed offline in epochs of 1-sec (Cooke et al., 2014; Moore, Vine, Cooke, Ring, \& Wilson, 2012; Neumann \& Thomas, 2011). Heart rate was corrected for artefacts and R-wave peaks were identified. The intervals between the successive R-waves peaks were calculated and instantaneous heart rate (beats per minute, BPM) was calculated as 6000/(R-R interval). Muscle activity was assessed by rectifying the EMG signal and averaging over 0.5-sec windows, such that the mean activity between 6.25 and 5.75 -sec prior to movement was used to calculate muscle activity 6-sec before movement, and so on (Cooke et al., 2014). phase until the point of contact (Cooke et al., 2014; Cooke et al., 2010; Moore et al., 2012). Average acceleration was calculated for the $\mathrm{x}, \mathrm{y}$, and z-axes. Besides impact velocity, Root Mean Square (RMS) jerk and smoothness on the z-axis were computed, as the z-axis is the main axis involved in the putting swing (Cooke et al., 2011; Maxwell et al., 2003).

Statistical analysis.

The cortical activity manipulation check was subjected to a 3 x 10 repeated measures analysis of variance (ANOVA): Condition (Left, Right, No-hand) x Homologous electrode pairs (T8-T7, P4-P3, P8-P7, F4-F3, F8-F7, C4-C3, FC2-FC1, FC6-FC5, CP2-CP1, CP6- 
CP5). The T7-Fz connectivity measure during preparation of the golf putt was subjected to a one-way ANOVA of Condition (Left, Right, No-hand). Cardiac and muscle activity were subjected to a 3 x 13 repeated measures ANOVA: Condition (Left, Right, No-hand) x Time Bin $(-6,-5,-4,-3,-2,-1,0,+1,+2,+3,+4,+5,+6)$. Golf putting kinematics and golf putting performance were both subjected to a one-way ANOVA of Condition (Left, Right, No-hand). Sphericity was checked and corrected using the Huynh-Feldt correction when necessary. Separate ANOVAs with Bonferroni corrections or polynomial trend analysis were performed when main effects or interactions were found. Effect sizes are reported as partial $\eta$ squared $\left(\eta_{\mathrm{p}}{ }^{2}\right)$. The statistical tests were performed using SPSS (IBM, version 25.0) computer software. Significance was set at $p=.05$ for all statistical tests. designs, Montoya \& Hayes, 2017) was used to test within-subject mediation effects on golf putting performance associated with left-hand and right-hand contractions. Mediators were individually tested and included EEG, EMG, ECG and kinematics (i.e., club head orientation, swing height and impact force). The mediation effect (B), standard error (BootSE) and 95\%

300 CI (low and high) were reported (Montoya \& Hayes, 2017).

\section{Results}

302

303

\section{Manipulation check}

The results revealed a main effect of Condition, $\mathrm{F}(2,42)=3.95, p=.027, \eta_{\mathrm{p}}{ }^{2}=.16$, with post-hoc analysis revealing a significantly lower asymmetry score for left-hand contractions compared with right-hand contractions ( $p=.015$, see Fig. 1). No significant effects were revealed for left-hand contractions compared with no-hand contractions ( $p$ $=.180)$ or right-hand contractions compared with no-hand contractions $(p=1.00)$. No main effect was found for Homologous electrode pairs, $\mathrm{F}(3.20,67.15)=0.93, p=.438, \eta_{\mathrm{p}}{ }^{2}=.04$. 


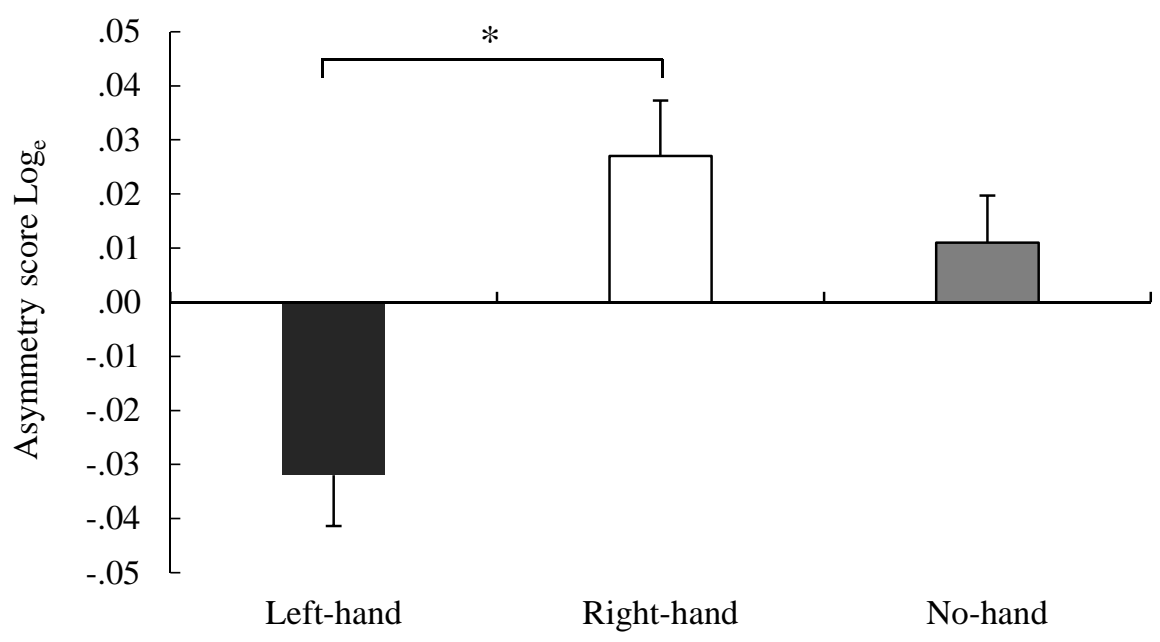

309

310 Fig. 1. Alpha power asymmetry score per condition. Asymmetry score was calculated by: right hemisphere -

311 left hemisphere (positive values represent higher right-hemisphere power and negative values represent higher

312 left-hemisphere power). Error bars represent standard error of the mean. $(* p<.05)$.

313 Cortical activity preceding golf putts

314 The results revealed a main effect of Condition, $\mathrm{F}(2,48)=122.5, p<.001, \eta_{\mathrm{p}}{ }^{2}=.84$.

315 Post-hoc tests revealed that left-hand contractions led to significantly lower T7-Fz

316 connectivity, than right-hand contractions $(p<.001)$ or no-hand contractions $(p<.001$, see

317 Fig. 2). Right-hand contractions revealed the opposite effect with significantly higher T7-Fz

318 connectivity compared to left-hand contractions $(p<.001)$ and no-hand contractions $(p$

$319<.001$, see Fig. 2). 


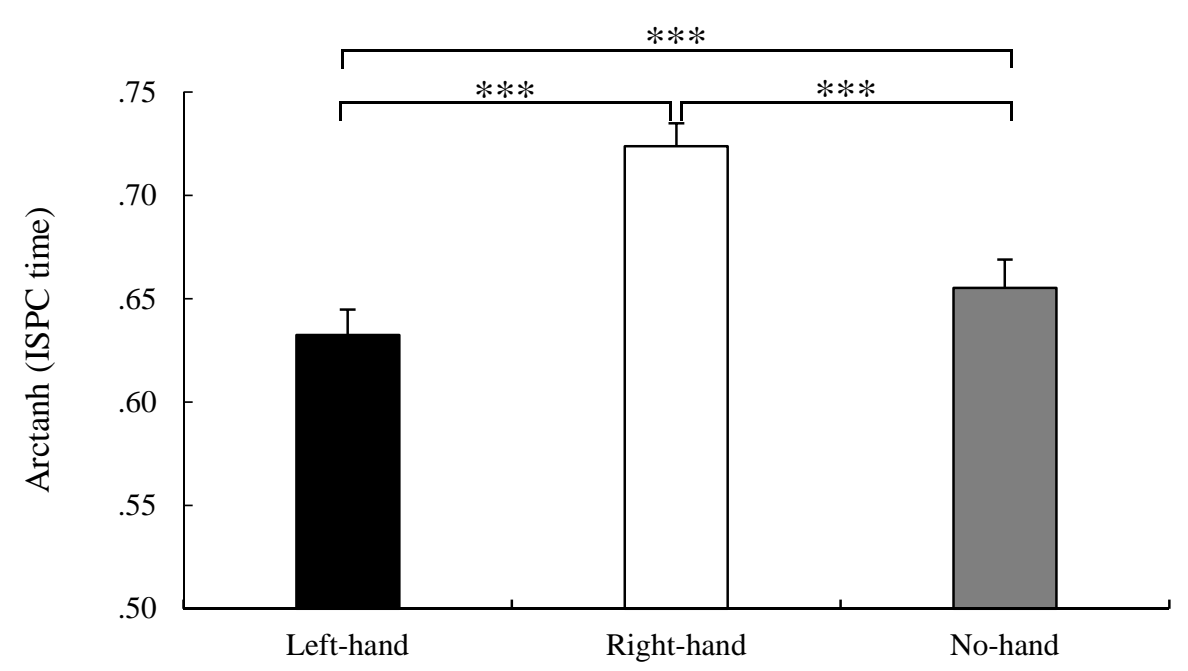

321 Fig. 2. T7-Fz ISPCtime connectivity during each condition and time bin. Error bars represent standard error of

322 the mean. $(* * \mathrm{p}<.001)$.

323 Muscle activity

No Condition x Time Bin interactions were evident for the extensor carpi radialis, $\left(\mathrm{F}(24,432)=1.15, p=.290, \eta_{\mathrm{p}}{ }^{2}=.06\right.$, or the flexor carpi ulnaris, $\mathrm{F}(24,480)=0.82, p=.715$, $\eta_{\mathrm{p}}{ }^{2}=.04$. A main effect of Time Bin was evident for the extensor carpi radialis, $\mathrm{F}(3.73,67.11)$ $=9.99, p<.001, \eta_{\mathrm{p}}{ }^{2}=.36$, and the flexor carpi ulnaris, $\mathrm{F}(4.18,83.61)=13.51, p<.001, \eta_{\mathrm{p}}{ }^{2}$ $=.40$. Post-hoc analysis revealed that for the extensor carpi radialis the variance for Time Bin was best described by a quadratic trend $\left(p<.001, \eta_{\mathrm{p}}{ }^{2}=.53\right)$, with a gradual increase of

330 activity until peak in activity during movement initiation (time zero), which quickly drops

331 back to baseline (see Fig. 3). For the flexor carpi ulnaris, variance for Time Bin was also best 332 described by a quadratic trend $\left(p<.001, \eta_{\mathrm{p}}{ }^{2}=.68\right)$, with similar trends to the extensor carpi 333 radialis (see Fig. 4). Main effects of Condition were not evident for the extensor carpi radialis,

$334 \mathrm{~F}(2,36)=1.74, p=.191, \eta_{\mathrm{p}}{ }^{2}=.09$, or the flexor carpi ulnaris, $\mathrm{F}(2,40)=0.69, p=.510, \eta_{\mathrm{p}}{ }^{2}$ $335=.03$. 


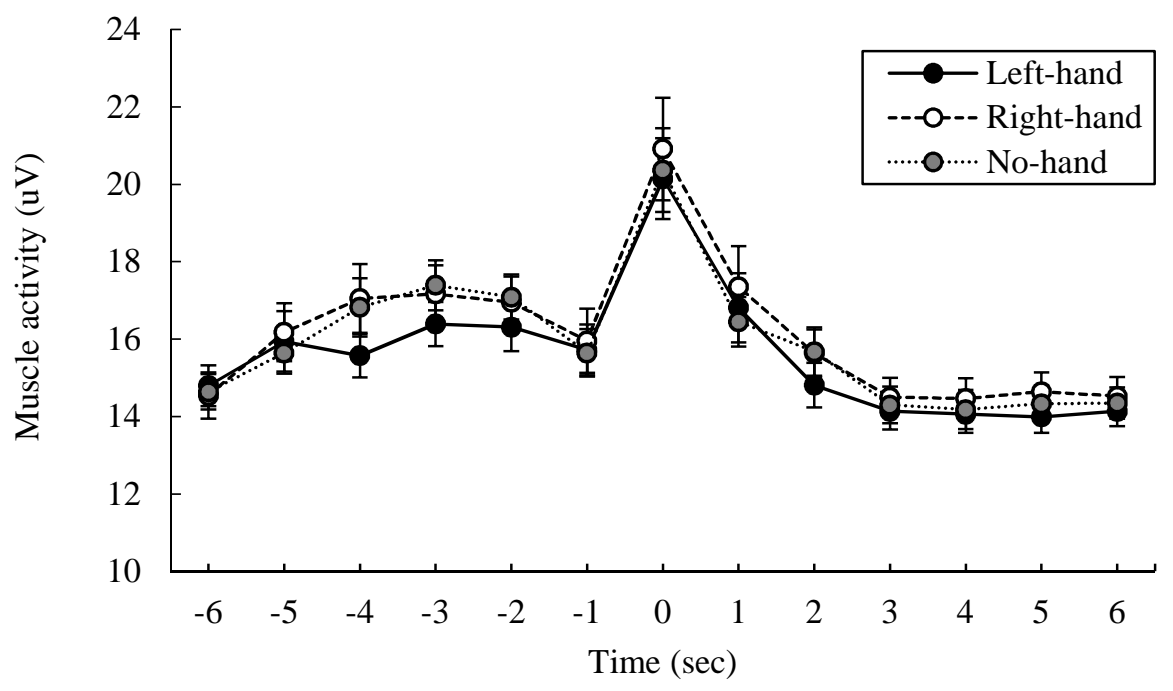

Fig. 3. Activity of the extensor carpi radialis in each condition over time. Error bars represent standard error of the mean.

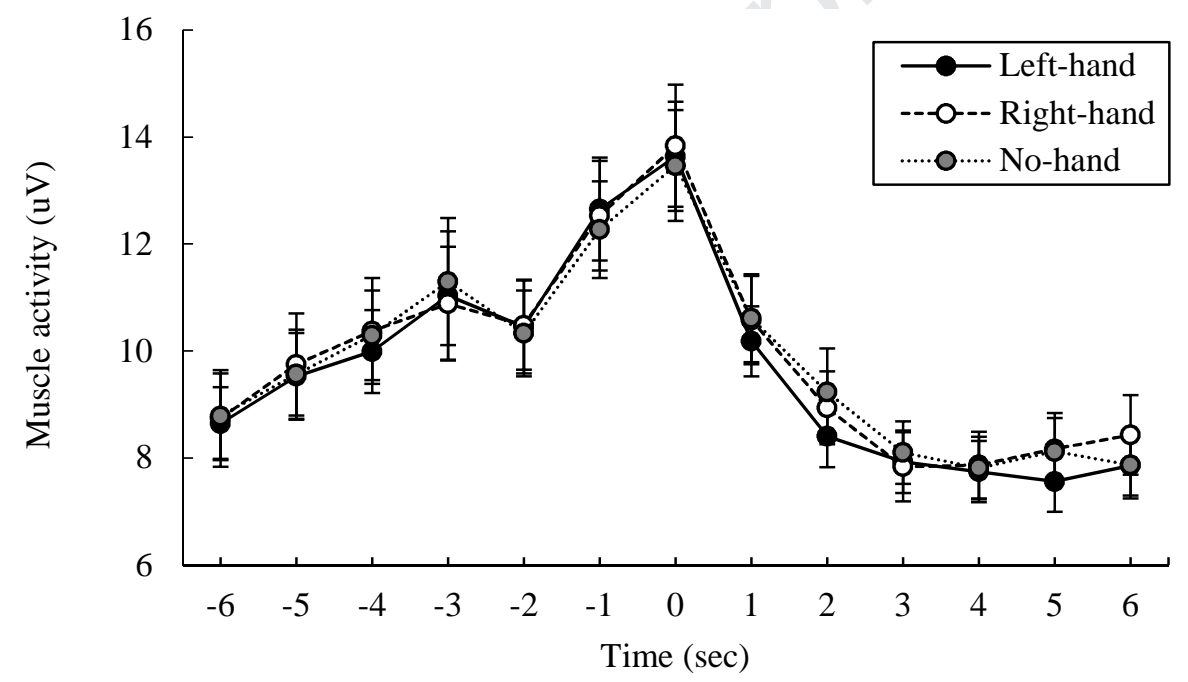

Fig. 4. Activity for of the flexor carpi ulnaris in each condition over time. Error bars represent standard error of

341 the mean.

\section{Cardiac activity}

The ECG analysis did not reveal a Condition $\mathrm{x}$ Time Bin interaction, $\mathrm{F}(24,567)=0.95$,

$344 p=.532, \eta_{\mathrm{p}}{ }^{2}=.04$, or a main effect of Condition, $\mathrm{F}(2,48)=0.62, p=.542, \eta_{\mathrm{p}}{ }^{2}=.03$. A main

345 effect of Time Bin was evident, $\mathrm{F}(1.57,37.61)=17.26, p<.001, \eta_{\mathrm{p}}{ }^{2}=.42$. Post-hoc analysis

346 revealed that heart rate differences over time was best described by a cubic trend $(p<.001$,

$\left.347 \eta_{\mathrm{p}}{ }^{2}=.56\right)$. Heart rate decreased during approximately 2 -sec preceding movement initiation

348 and then gradually retrurned to baseline in the 6-sec after movement initiation (see Fig. 5). 


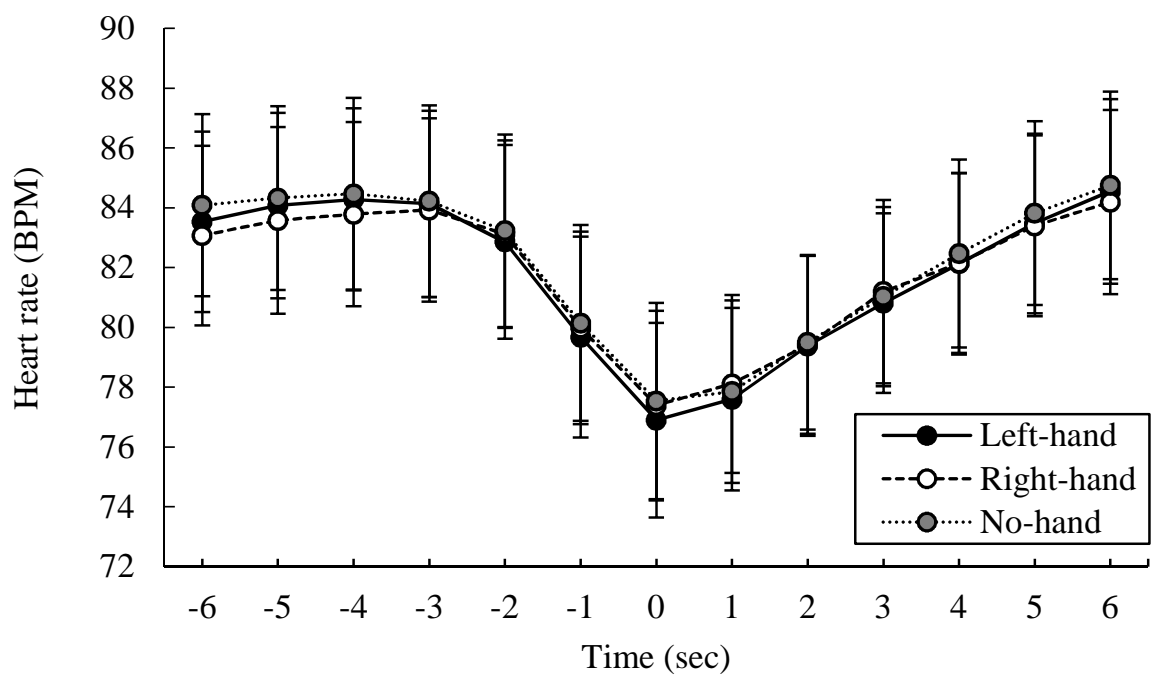

350 Fig. 5. Heart rate in each condition over time (6-sec before until 6-sec after movement initiation). Error bars represent standard error of the mean.

\section{Golf kinematics}

No differences were evident between conditions for any of the kinematic measures: acceleration on the $\mathrm{x}$-axis, $\mathrm{F}(2,48)=2.60, p=.085, \eta_{\mathrm{p}}^{2}=.10$; acceleration on the $\mathrm{y}$-axis, $\mathrm{F}(1.59,38.26)=0.65, p=.493, \eta_{\mathrm{p}}^{2}=.03$; acceleration on the $\mathrm{z}$-axis, $\mathrm{F}(2,44)=0.55, p=.581$, $\eta_{\mathrm{p}}{ }^{2}=.02$; impact speed, $\mathrm{F}(1.52,36.39)=0.25, p=.718, \eta_{\mathrm{p}}{ }^{2}=.01 ;$ RMS jerk, $\mathrm{F}(2,46)=0.31, p$ $=.738, \eta_{\mathrm{p}}^{2}=.01 ;$ smoothness, $\mathrm{F}(1.59,38.03)=0.46, p=.592, \eta_{\mathrm{p}}{ }^{2}=.02$.

\section{Golf putting performance}

No differences were evident between conditions for mean radial error, $\mathrm{F}(2,48)=1.75$, $p=.184, \eta_{\mathrm{p}}^{2}=.07$.

\section{Mediation analysis}

Mediation analyses were used to examine whether EEG, EMG, ECG or kinematics

mediated the relationship between hand contractions and golf putting performance (mean radial error). Although there was no significant difference in performance between the different hand contraction conditions, there was a significant indirect effect of hand

367 following left-hand versus right-hand contractions were mediated by the changes in EEG T7- 
Fz connectivity induced by these protocols, $\mathrm{B}=-12.41$, BootSE $=4.12,95 \% \mathrm{CI}[-21.07,-$

4.94]. The other mediators did not reveal significant indirect effects on performance.

\section{Discussion}

The present study was conducted to examine whether pre-performance unilateral hand contraction protocols influence verbal-analytical engagement in motor performance. A

373 repeated measures crossover design was adopted, measuring psychophysiological markers

374 (neural, cardiovascular and muscular) and performance (distance from the target and movement kinematics) of a golf putting task that was completed immediately after performing a hand contraction protocol (left, right and no-hand). During the hand contraction protocols, measures of alpha power spectra between homologous electrode pairs were computed as a manipulation check to determine whether hand contractions caused different hemispheric activation.

The manipulation check revealed a significant difference in hemispheric asymmetry between left-hand and right-hand contraction protocols, with the left-hand contraction protocol resulting in more right-hemisphere activity and the right-hand contraction protocol resulting in higher left-hemisphere activity (see Fig. 1). These findings are consistent with previous studies (Gable et al., 2013; Harmon-Jones, 2006; Peterson et al., 2008).

Our study is the first to include a no-hand contractions, which makes it possible to compare the effect of left-hand and right-hand contractions relative to no contractions.

387 Asymmetry during the no-hand contraction protocol was not significantly different from

388 either contraction condition, which suggests that hand contractions did not create different asymmetry compared to no-hand contractions. However, hand contractions did achieve different asymmetry compared to each other. The slight rightward bias evident during the no-

391 hand condition is in line with previous studies revealing that right-handedness is related to a 
392 bias to rightward hemisphere asymmetry (greater left-hemisphere activity) for resting state

393 alpha power (e.g., Ocklenburg et al., 2019).

394 As hypothesized, a lower level of T7-Fz connectivity during preparation for putts was

395 revealed after left-hand contractions, compared to right-hand and no-hand contractions. The

396 opposite effect was found for right-hand contractions, revealing higher T7-Fz connectivity

397 compared to left-hand and no-hand contractions. Previous studies have suggested that lower

398 T7-Fz connectivity reflects less verbal-analytical engagement in movements (e.g., Deeny et

399 al., 2003; Gallicchio et al., 2016; Zhu et al., 2011). Left-hand contractions in the present

400 study may therefore have lowered T7-Fz connectivity and reduced verbal-analytical

401 engagement in the putting task, compared to right-hand and no-hand contractions.

402 Although there was no significant effect of hand contractions on golf putting

403 performance, ${ }^{3}$ mediation analysis suggested that hand contractions influenced $\mathrm{T} 7-\mathrm{Fz}$

404 connectivity, which in turn influenced performance. Beckmann et al. (2013) and Gröpel and

405 Beckmann (2017) speculated that top-down verbal-analytical control processes are the

406 mechanism by which hand contractions influence performance under pressure. Many

407 explanations of skill failure, such as the theory of reinvestment (Masters, 1992; see Masters

$408 \&$ Maxwell, 2008 for a review), suggest that attempts to consciously control movements

409 (characterised by verbal-analytical processing), can disrupt normally efficient motor

410 behaviours. Given the hypothesised link between T7-Fz connectivity and conscious verbal

411 engagement of movement, our mediation findings provide some support for their speculation.

412 Although the hand contraction protocols clearly influenced neurophysiological

413 activity, their effects did not extend to the cardiac, muscular or kinematic measures. There

${ }^{3}$ It is acceptable to conduct mediation analysis when there is no significant effect of the independent variable (hand contractions) on the dependent variable (golf putting performance) (see e.g., Kenny, Kashy, \& Bolger, 1998). 
414 were no condition effects for these variables and there were no mediational effects to

415 implicate any of these variables in the relationship between hand contractions and

416 performance. From a theoretical perspective it makes sense that neural measures should be

417 more sensitive to the effects of hand contraction protocols than peripheral measures such as

418 heart rate, because verbal-analytic processes originate from the brain, and any effects they

419 might have on the heart and muscles would be always be secondary. Any effects of

420 psychological processes on cardiac and muscular activity could also have been masked by

421 any physical strain on these variables caused by the golf putting task (e.g., standing posture,

422 swinging arms, etc.).

423 Despite the indirect effect of hand contractions on performance through $\mathrm{T} 7-\mathrm{Fz}$

424 connectivity, there were no significant performance differences between the different hand

425 contraction protocols. Our participants only performed 130 trials prior to the first hand

426 contraction condition, so they remained relatively inexperienced novices with high inter and

427 intra person performance variability that may have camouflaged any subtle (direct) hand

428 contraction effects. A more cognitively challenging task may reveal performance differences.

429 Zhu et al (2015) also manipulated T7-Fz coherence, using real versus sham tDCS, and also

430 failed to find an effect on golf putting performance alone. However, Zhu et al. (2015) did

431 report a differential effect on golf putting performance under dual-task load (e.g., backwards

432 counting). Alternatively, replicating the experiment with more experienced performers could

433 also increase the likelihood of performance differences. For example, the theory of

434 reinvestment (Masters \& Maxwell, 2008) argues that verbal-analytic engagement (e.g., right-

435 hand contractions) would be more detrimental to the performance of autonomous experts than

436 cognitive novices. Effects of condition on the cardiac, muscular and kinematic measures

437 would also be more likely with experienced performers for the same reasons. 
A limitation of this study is that we did not control force of grip used by participants

during the hand contraction protocol. Consequently, differences in hemisphere asymmetry

440 might have been a function of effort or strength. For example, Hirao and Masaki (2018)

441 showed that force and duration of left-hand contractions had differential effects on

442 hemisphere activity. Additionally, a requirement to achieve a specific force during

443 contractions may require more cognitive resources (e.g., Derosière et al., 2014; Hirao \&

444 Masaki, 2018). One solution might simply be to measure grip force and include it as a studies.

Another limitation is that we were unable to determine the longevity of the hand contractions with respect to their effect on cortical activity. Studies suggest that the effects of hand contraction protocols last at least 15-min (e.g., Baumer, Munchau, Weiller, and Liepert (2002). Participants in our study completed 25 trials over approximately a 6-min duration, so it is likely that the effects remained. However, there is little doubt that further research is needed to gain greater understanding of the timecourse of hand contraction effects. contractions lower verbal-analytical engagement in motor planning of a golf putting task. The additional markers (ECG, EMG, kinematics and performance) did not, however, provide supporting evidence of this effect. These secondary markers may have been insufficiently

457 sensitive to reveal the brain's influence over the body. Nevertheless, it appears that the body 458 (the hands) influenced the brain! 
461 Babiloni, C., Infarinato, F., Marzano, N., Iacoboni, M., Dassu, F., Soricelli, A., . . Del

462

463

464

465

466

467

468

469

470

471

472

473

474

475

476

477

478

479

480

481

482

483

484

Percio, C. (2011). Intra-hemispheric functional coupling of alpha rhythms is related to golfer's performance: a coherence EEG study. International Journal of Psychophysiology, 82(3), 260-268. https://doi.org/10.1016/j.ijpsycho.2011.09.008

Baumer, T., Munchau, A., Weiller, C., \& Liepert, J. (2002). Fatigue suppresses ipsilateral intracortical facilitation. Experimental Brain Research, 146, 467-473. https://doi.org/10.1007/s00221-002-1202-X

Beckmann, J., Gröpel, P., \& Ehrlenspiel, F. (2013). Preventing motor skill failure through hemisphere-specific priming: cases from choking under pressure. Journal of Experimental Psychology General, 142(3), 679-691. https://doi.org/10.1037/a0029852

Cohen, M. X. (2014). Analyzing neural time series data: Theory and practice. Cambridge, MA: MIT Press.

Cooke, A., Kavussanu, M., Gallicchio, G., Willoughby, A., McIntyre, D., \& Ring, C. (2014). Preparation for action: Psychophysiological activity preceding a motor skill as a function of expertise, performance outcome, and psychological pressure. Psychophysiology, 51, 374-384. https://doi.org/10.1111/psyp.12182

Cooke, A., Kavussanu, M., McIntyre, D., Boardley, I. D., \& Ring, C. (2011). Effects of competitive pressure on expert performance: Underlying psychological, physiological, and kinematic mechanisms. Psychophysiology, 48, 1146-1156. https://doi.org/10.1111/j.1469-8986.2011.01175.x

Cooke, A., Kavussanu, M., McIntyre, D., \& Ring, C. (2010). Psychological, muscular and kinematic factors mediate performance under pressure. Psychophysiology, 47(6), 1109-1118. https://doi.org/10.1111/j.1469-8986.2010.01021.x 
Corcoran, A. W., Alday, P. M., Schlesewsky, M., \& Bornkessel-Schlesewsky, I. (2018). Toward a reliable, automated method of individual alpha frequency (IAF) quantification. Psychophysiology, 55(7), 1-21. https://doi.org/10.1111/psyp.13064

Cross-Villasana, F., Gropel, P., Doppelmayr, M., \& Beckmann, J. (2015). Unilateral lefthand contractions produce widespread depression of cortical activity after their execution. PLoS One, 10(12), e0145867. https://doi.org/10.1371/journal.pone.0145867

Davis, N. J. (2013). Neurodoping: brain stimulation as a performance-enhancing measure. Sports Medicine, 43(8), 649-653. https://doi.org/10.1007/s40279-013-0027-z

Deeny, S. P., Hillman, C. H., Janelle, C. M., \& Hatfield, B. D. (2003). Cortico-cortical communication and superior performance in skilled marksmen: An EEG coherence analysis. Journal of Sport and Exercise Psychology, 25, 188-204. https://doi.org/10.1123/jsep.25.2.188

Delorme, A., \& Makeig, S. (2004). EEGLAB: an open source toolbox for analysis of singletrial EEG dynamics including independent component analysis. Journal of Neuroscience Methods, 134, 9-21.

Derosière, G., Alexandre, F., Bourdillon, N., Mandrick, K., Ward, T. E., \& Perrey, S. (2014). Similar scaling of contralateral and ipsilateral cortical responses during graded unimanual force generation. Neuroimage, 85, 471-477. https://doi.org/10.1016/j.neuroimage.2013.02.006

Gable, P. A., Poole, B. D., \& Cook, M. S. (2013). Asymmetrical hemisphere activation enhances global-local processing. Brain and Cognition, 83(3), 337-341. https://doi.org/10.1016/j.bandc.2013.09.012

Gallicchio, G., Cooke, A., \& Ring, C. (2016). Lower left temporal-frontal connectivity characterizes expert and accurate performance: High-alpha T7-Fz connectivity as a 
marker of conscious processing during movement. Sport, Exercise, and Performance Psychology, 5(1), 14-24. https://doi.org/http://dx.doi.org/10.1037/spy0000055

512 Grefkes, C., Eickhoff, S. B., Nowak, D. A., Dafotakis, M., \& Fink, G. R. (2008). Dynamic

513

514

515

516 intra- and interhemispheric interactions during unilateral and bilateral hand movements assessed with fMRI and DCM. Neuroimage, 41(4), 1382-1394. https://doi.org/10.1016/j.neuroimage.2008.03.048

Gröpel, P., \& Beckmann, J. (2017). A pre-performance routine to optimize competition performance in artistic gymnastics. The Sport Psychologist, 31(2), 199-207. https://doi.org/10.1123/tsp.2016-0054

Harmon-Jones, E. (2006). Unilateral right-hand contractions cause contralateral alpha power suppression and approach motivational affective experience. Psychophysiology, 43(6), 598-603. https://doi.org/10.1111/j.1469-8986.2006.00465.x

Hirao, T., \& Masaki, H. (2018). Effects of unilateral hand contraction on the persistence of hemispheric asymmetry of cortical activity. Journal of Psychophysiology, 1-8. https://doi.org/10.1027/0269-8803/a000215

Jaspers, H. H. (1958). The ten twenty electrode system of the International Federation. Electroencephalography and Clinical Neurophysiology, 10, 371-375.

Kenny, D. A., Kashy, D. A., \& Bolger, N. (1998). Data analysis in social psychology. Handbook of social psychology, 1, 233-265.

Lachaux, J. P., Rodriguez, E., Martinerie, J., \& Varela, F. J. (1999). Measuring phase synchrony in brain signals. Human Brain Mapping, 8(4), 194-208. https://doi.org/10.1002/(SICI)1097-0193(1999)8:4<194::AID-HBM4>3.0.CO;2-C

Landers, D. M., Petruzzello, S. J., Salazar, W., Crews, D. J., Kubitz, K. A., Gannon, T. L., \& Han, M. (1991). The influence of electrocortical biofeedback on performance in pre- 
elite archers. Medicine \& Science in Sports \& Exercise. https://doi.org/http://dx.doi.org/10.1249/00005768-199101000-00018

Lohse, K. R., Sherwood, D. E., \& Healy, A. F. (2010). How changing the focus of attention affects performance, kinematics, and electromyography in dart throwing. Human Movement Science, 29(4), 542-555. https://doi.org/10.1016/j.humov.2010.05.001

Lopez-Calderon, J., \& Luck, S. J. (2014). ERPLAB: an open-source toolbox for the analysis of event-related potentials. Frontiers in Human Neuroscience, 8. https://doi.org/10.3389/fnhum.2014.00213

Makeig, S., Bell, A. J., Jung, T. P., \& Sejnowski, T. J. (1996). Independent component analysis of electroencephalographic data. In D. Touretzky, M. Mozer, \& M. Hasselmo (Eds.), Advances in neural information processing systems (Vol. 8, pp. 145-151). Cambridge, MA: MIT Press.

Malhotra, N., Poolton, J. M., Wilson, M. R., Omuro, S., \& Masters, R. S. W. (2015). Dimensions of movement specific reinvestment in practice of a golf putting task. Psychology of Sport and Exercise, 18, 1-8. https://doi.org/10.1016/j.psychsport.2014.11.008

Masters, R. S. W. (1992). Knowledge, knerves and know-how: The role of explicit versus implicit knowledge in the breakdown of a complex motor skill under pressure. British Journal of Psychology, 83(3), 343-358. https://doi.org/10.1111/j.20448295.1992.tb02446.x

Masters, R. S. W., Eves, F. F., \& Maxwell, J. (2005, 15-19 August 2005). Development of a Movement Specific Reinvestment Scale. Paper presented at the ISSP 11th World Congress of Sport Psychology, Sydney, Australia. 
557

558

559

560

561

562

563

564

565

566

567

568

569

570

571

572

573

574

575

576

577

578

579

580

581

Masters, R. S. W., \& Maxwell, J. P. (2008). The theory of reinvestment. International Review of Sport and Exercise Psychology, 1(2), 160-183. https://doi.org/10.1080/17509840802287218

Masters, R. S. W., Poolton, J. M., Maxwell, J. P., \& Raab, M. (2008). Implicit motor learning and complex decision making in time-constraints environments. Journal of Motor Behavior, 40(1), 71-79. https://doi.org/10.3200/JMBR.40.1.71-80

Maxwell, J. P., Masters, R. S. W., \& Eves, F. F. (2003). The role of working memory in motor learning and performance. Consciousness and Cognition, 12(3), 376-402. https://doi.org/10.1016/s1053-8100(03)00005-9

Montoya, A. K., \& Hayes, A. F. (2017). Two-condition within-participant statistical mediation analysis: A path-analytic framework. Psychological Methods, 22(1), 6-27. https://doi.org/10.1037/met0000086

Moore, L. J., Vine, S. J., Cooke, A., Ring, C., \& Wilson, M. R. (2012). Quiet eye training expedites motor learning and aids performance under heightened anxiety: the roles of response programming and external attention. Psychophysiology, 49(7), 1005-1015. https://doi.org/10.1111/j.1469-8986.2012.01379.x

Neumann, D. L., \& Thomas, P. R. (2008). A camera-based scoring system for evaluating performance accuracy during a golf putting task. Behavior Research Methods, 40(3), 892-897. https://doi.org/10.3758/brm.40.3.892

Neumann, D. L., \& Thomas, P. R. (2009). The relationship between skill level and patterns in cardiac and respiratory activity during golf putting. International Journal of Psychophysiology, 72(3), 276-282. https://doi.org/10.1016/j.ijpsycho.2009.01.001

Neumann, D. L., \& Thomas, P. R. (2011). Cardiac and respiratory activity and golf putting performance under attentional focus instructions. Psychology of Sport and Exercise, 12(4), 451-459. https://doi.org/10.1016/j.psychsport.2011.02.002 
582

583

584

585

586

587

588

589

590

591

592

593

594

595

596

597

598

599

600

601

602

603

604

Ocklenburg, S., Friedrich, P., Schmitz, J., Schluter, C., Genc, E., Gunturkun, O., . . . Grimshaw, G. (2019). Beyond frontal alpha: investigating hemispheric asymmetries over the EEG frequency spectrum as a function of sex and handedness. Laterality, 24(5), 505-524. https://doi.org/10.1080/1357650X.2018.1543314

Oldfield, R. C. (1971). The assessment and analysis of handedness: the Edinburgh inventory. Neuropsychologia, 9(1), 97-113. https://doi.org/10.1016/0028-3932(71)90067-4

Peterson, C. K., Shackman, A. J., \& Harmon-Jones, E. (2008). The role of asymmetrical frontal cortical activity in aggression. Psychophysiology, 45(1), 86-92. https://doi.org/10.1111/j.1469-8986.2007.00597.x

Radlo, S. J., Steinberg, G. M., Singer, R. N., Barba, D. A., \& Melnikov, A. (2002). The influence of an attentional focus strategy on alpha brain wave activity, heart rate and dart-throwing performance. International Journal of Sport Psychology.

Schiff, B. B., Guirguis, M., Kenwood, C., \& Herman, C. P. (1998). Asymmetrical hemispheric activation and behavioral persistence: effects of unilateral muscle contractions. Neuropsychology, 12(4), 526-532. https://doi.org/10.1037/08944105.12.4.526

Smith, M. E., McEvoy, L. K., \& Gevins, A. (1999). Neurophysiological indices of strategy development and skill acquisition. Cognitive Brain Research, 7, 389-404. https://doi.org/10.1016/S0926-6410(98)00043-3

Snyder, A. W., Mulcahy, E., Taylor, J. L., Mitchell, D. J., Sachdev, P., \& Gandevia, S. C. (2003). Savant-like skills exposed in normal people by suppressing the left frontotemporal lobe. Journal of Integrative Neuroscience, 2(2), 149-158. https://doi.org/10.1142/S0219635203000287 
605

606

607

608

609

610

611

612

613

614

615

616

617

618

619

620

621

622

623

Vance, J., Wulf, G., Tollner, T., McNevin, N., \& Mercer, J. (2004). EMG activity as a function of the performer's focus of attention. Journal of Motor Behavior, 36(4), 450459. https://doi.org/10.3200/JMBR.36.4.450-459

Wolf, S., Brölz, E., Keune, P. M., Wesa, B., Hautzinger, M., Birbaumer, N., \& Strehl, U. (2015). Motor skill failure or flow-experience? Functional brain asymmetry and brain connectivity in elite and amateur table tennis players. Biological Psychology, 105, 95105. https://doi.org/http://dx.doi.org/10.1016/j.biopsycho.2015.01.007

Zachry, T., Wulf, G., Mercer, J., \& Bezodis, N. (2005). Increased movement accuracy and reduced EMG activity as the result of adopting an external focus of attention. Brain Research Bulletin, 67(4), 304-309. https://doi.org/10.1016/j.brainresbull.2005.06.035

Zhu, F. F., Poolton, J. M., Wilson, M. R., Maxwell, J. P., \& Masters, R. S. W. (2011). Neural co-activation as a yardstick of implicit motor learning and the propensity for conscious control of movement. Biological Psychology, 87(1), 66-73. https://doi.org/10.1016/j.biopsycho.2011.02.004

Zhu, F. F., Yeung, A. Y., Poolton, J. M., Lee, T. M. C., Leung, G. K. K., \& Masters, R. S. W. (2015). Cathodal Transcranial Direct Current Stimulation over left dorsolateral prefrontal cortex area promotes implicit motor learning in a golf putting task. Brain Stimulation, 8(4), 784-786. https://doi.org/10.1016/j.brs.2015.02.005 
Highlights:

- The effects of unilateral hand contractions during motor performance was investigated

- Unilateral hand contractions influenced the verbal-analytical engagement in motor planning

- Left-hand contractions caused lower verbal-analytical engagement in motor planning

- It appears that the body (the hands) influenced the brain! 


\section{Declaration of interests}

$\bigotimes$ The authors declare that they have no known competing financial interests or personal relationships that could have appeared to influence the work reported in this paper.

$\square$ The authors declare the following financial interests/personal relationships which may be considered as potential competing interests: 